\title{
After Strict Comparison, the Conclusion of Double Slit Test Is in Conflict With the Mathematical Model
}

\author{
Xia Ruhuai ( $\sim 2969169228 @ q q . c o m)$ \\ $\mathrm{N} / \mathrm{A}$
}

\section{Research Article}

Keywords: double-slit interference, Michelson-Morley experiment, polarization, light waves, wave-particle duality, superposition, causality, the theory of relativity, antimatter, quantum model, to observe the change as a result, the Schrodinger's cat, matter waves, the speed of light is changeless, Huygens' principle, the Doppler effect, the redshift, regression, Hubble's law, the expansion of the universe, dark matter and dark energy, Kepler's laws of planetary, Thomas Yang, bandwidth, quantum segmentation, quantum multiplication, standing wave, fiber optic communication, modulation effects

Posted Date: September 16th, 2021

DOI: https://doi.org/10.21203/rs.3.rs-744658/v4

License: (c) (1) This work is licensed under a Creative Commons Attribution 4.0 International License. Read Full License 


\section{Abstract}

The double slit test, as it is known, results in a series of alternating streaks of light and dark, presumed to be caused by interfering light waves. Careful comparison of experimental results and mathematical models reveals that the plausible theory of light-wave interference contains many fatal flaws. For example, the mathematical model does not have a mechanism for regularly producing multiple dark streaks. In practice, the spot where the dark streaks should appear is the brightest spot. Since only the electron radiates photons outward as it lowers its energy and returns to its ground state, the photon waves generated in the slit (possibly a vacuum) are of unknown origin. When light waves interfere with each other, photons can be infinitely subdivided and multiplied; After the head of the photon reaches the screen, the rest of the photon can still participate in interference; The effects of reflected light waves that most satisfy the interference conditions are not shown in the fringe. The important influence of polarization direction is not considered in the interference condition. In the causality test, the photon that has collapsed into a particle should be retested with a double slit test. In the causality test, the photon that has collapsed into a particle should be retested with a double slit test. The phenomenon of "observation determines outcome" has also been observed. When the phase is shifted $\pi$ or the signal is reversed, it becomes a negative wave that cancels itself out. The transmittance of light waves through a double-slit device should not be so weak. As a fundamental physical property, waves must have bandwidth, but light and quantum only have a single frequency, which does not conform to the Fourier transform principle that waves must follow. Light waves in optical fiber communication must produce modulation effect, which has not been shown in reality. If the light waves from different slits interfere with each other, a mask similar to a two-slit device will not allow the lithographer to work properly. A photon whose size is much smaller than the wave length does not represent the whole wave but only represents a sample of the wave, but the corresponding physical properties are not shown. A pair of stars orbiting each other without changing color only proves that redshift is impossible but not that the speed of light is constant. Due to the late birth of radio technology, many physical properties of electromagnetic waves were not included in the light and matter waves proposed earlier. The results of the double slit test only prove that the simple quantum particle model is incorrect but do not provide evidence that the wave model is correct.

\section{Background}

\section{The basic formula of quantum is as follows:}

$E=h^{\star} f$

Where $E$ : quantum energy. $h$ : Planck constant. $f$. Frequency.

Note: Planck constant $h$ is a general constant. For any matter, the Planck constant $h$ is constant. Frequency $f$ is a special constant. Different kinds of matter have their own special frequency constant $f$, that is, each quantum frequency $f$ has and only has one unique constant. 


\subsection{If the conclusion of the double slit test is correct, the light wave can be subdivided and multiplied indefinitely}

In Fig. 3, on the double-slit test device, the left slit is SL, and the right slit is SR. Without loss of generality, a certain light wave passing through the slit $S L$ is named $L_{A}$, and the wave group of $n$ light waves passing through the other slit SR in chronological order is named $L_{B}$, where the value $n$ is a non-zero and uncapped natural number. According to the principle that light waves from different slits must interfere with each other, each light wave $L_{A}$ interferes with each light wave in a group of $n$ light waves, $L_{B}$. $L_{A}$ and $L_{B}$ must produce $n$ interference points, which form streaks on the screen. This leads to the following absurd conclusions: If $\mathrm{L}_{\mathrm{A}}$ is a group of $m$ light waves passing through the slit in chronological order, the resulting interference points are the product $m * n$. A single light wave $L_{A}$ can interfere with each member of the $L_{B}$ containing any number of light waves, that is, each photon $L_{A}$ can be subdivided into any number of parts. According to the principle of double slit interference, a new photon is born at each interference point. A group of $m$ light waves in one slit and a group of $n$ light waves in another slit can interfere with $m * n$ light waves. Each quantum can be multiplied into an infinite number.

Also, see Fig. 3. The bulge part of the light wave must always precede its interference region; that is, the head of the light wave always arrives at the screen before its interference region. When the bulge of the light wave reaches the screen, it is imprinted with the label of the photon, indicating that it has been used by the screen. At the moment that the distance from the screen to the top of the interference point still has a distance, the distance is proportional to $|G|$. According to the conclusion of the double-slit interference test, the following incorrect inference can be derived: The light wave that loses its head can continue to produce interference phenomena. The screen consumes only part of the light wave but not all of the light wave. This clearly contradicts the fundamental principles of quantum mechanics. A light wave without a head cannot have a residual part that interferes with an infinite number of waves coming from another slit. Even if light is a wave, because quanta are indivisible, the rest of the wave head must collapse into nothing when it reaches the screen.

\subsection{The mathematical model of light wave interference is not rigorous, and the mechanism of dark fringe is not clear}

The mathematical model of the interference principle shown in Fig. 3 is inconsistent with the actual results of the double-slit test shown in Fig. 4(a). According to Fig. 3 of the mathematical model, the only place that produces dark stripes is located in the center of the two targets, TS and TR, and the rest places do not produce dark stripes. Compared with the experimental results shown in Fig. 4(a), the center of the fringe is not only the dark fringe, but the brightest part of the whole fringe. In addition, there are multiple dark stripes that appear regularly in other positions.

Interference conditions for waves include frequency and phase. When these two crests meet, they create superposition and they inevitably create negative superposition of the trough. This is actually resonance, at the point of resonance, the result of the superposition of $m$ light waves in accordance with the phase 
conditions is to increase the intensity of light up to $m$ times. When the peaks and troughs meet, they cancel each other out, and the corresponding physical phenomenon is the annihilation of light waves and negative light waves. If light waves are matter then negative light waves are antimatter.

The mathematical model of the principle of interference shown in Figure 3 is a schematic diagram. If there is a significant gap between photons, the light is dim to almost invisible. When the light is bright enough that there is no visible gap between the waves, each photon will overlap with countless others to varying degrees. Dark streaks are impossible according to this model.

What results from the interference effect of water waves are standing waves that are stationary and cannot propagate. Similarly, light waves should obey the same physical properties, and the light produced by interference should also be static light that cannot propagate and cannot reach the screen.

Similar to monochromatic light, water waves have a single frequency. Composite light contains "light waves" of different wavelengths. The conditions under which light waves interfere with each other and produce dispersion effects are similar. However, the dispersion phenomenon is not present in the results of the double slit test.

Compared with the interference results of water waves, the bright fringe corresponds to the crest of the water fringe, and the dark fringe corresponds to the part between the crest. As a wave travels, the width of the crests is almost constant, but the width between the crests expands rapidly. In contrast, the double-slit test results show that with increasing distance $Y$ in Fig. 3, the width $E$ of the bright fringe in Fig. 4 expands rapidly, but the width e of the dark fringe is almost constant. When $Y$ is far enough away, $E$ is much larger than e. However, according to Fig. 3, E and e should be similar rather than vastly different.

A water wave consists of multiple waves and the distance between the waves is the wavelength. A wavelength contains an untold number of "light waves" because "light waves" are much smaller in size than the wavelength. The number of "light waves" contained in a wavelength determines the brightness of a beam. The denser the wave, the brighter the beam, and the thinner the wave, the darker the beam.

As shown in Fig. 5, when the attitude of double-slit device $S 2$ is rotated from Z0 to Z1, the fringes of the double-slit test do not change. This result is inconsistent with existing mathematical and physical models of waves.

As shown in Fig. 6 , the divergence angle $\beta$ of the slit corresponding to the total length of the fringe in the double-slit "interference" experiment is very small, which is difficult to understand if light is a wave.

\subsection{Disaster for light and matter wave theory, superposition of antimatter and matter}

In Fig. 1, without loss of generality, the mathematical expression for waves can be set as $u_{1}(t)=A \sin (2 \pi f$ $\left.t+\theta_{0}\right)$. Where $A$ is the amplitude, $f$ is the frequency, $t$ is time, $\theta$ is the phase, and $\theta_{0}$ is the initial phase. If the phase of light wave $u_{1}(t)$ is delayed or advanced by an odd multiple of $\pi$, its mathematical expression becomes: 
$u_{2}(t)=A \sin \left(2 \pi f t+\theta_{0} \pm(2 k+1) \pi\right)=-u_{1}(t)$

where $k$ is an arbitrary integer. Because $u_{1}(t)+u_{2}(t)=0, u_{1}(t)$ and $u_{2}(t)$ are a pair of opposite waves. Fig. 2 shows a circuit that produces opposite waves. When the opposite waves meet, they cancel each other out. $u_{1}(t)$ and $u_{2}(t)$ can annihilate each other. The mathematical model of wave is shown in Fig. 1. Using any moment as a reference, because the phase of the wave changes periodically over time, the polarity of the wave changes every half period. The role of waves fluctuates between positive and negative waves over time. The region $X$ in Fig. 1, which has a length of half a period, is both the negative half period of the positive wave and the positive half period of the negative wave. For any initial phase $\theta_{0}$, the phases of

$u_{1}(t)$ and $u_{2}(t)$ are always opposite. Thus, for different references, any point on the wave is both a positive and a negative wave. Obviously, this conclusion applies to electromagnetic wave, light wave and matter wave. For man-made electromagnetic waves, the key feature is waves, and it is easy to understand that positive and negative waves cancel each other out. But the key feature of matter and light is objective matter, and negative waves are antimatter. If light and matter are waves, matter and antimatter change roles periodically, and matter is also antimatter. Light and negative light change roles periodically, and light is also negative.

\subsection{The effect of medium is opposite to wave and light, which reinforces doubts about the light-wave model}

Without the need for any medium, including the aether, light can travel as fast as it can to infinity in an absolute vacuum with no matter at all. Any matter it encounters is an obstacle to the transmission of light. Although it is allowed to propagate in it, any transparent medium reduces not only the speed of light but also the distance it travels. The mass density of transparent medium is closely related to the allowable speed and distance of light propagation. The greater the mass density is, the slower the speed of light and the shorter the propagation distance in the medium. The smaller the mass density is, the faster the speed of light and the longer the propagation distance in the medium. Different wavelengths of light have different refractive indices in the same medium.

Mechanical waves can only travel through the medium, which is an indispensable condition, not an obstacle, to the propagation of mechanical waves. In general, the material density of the medium is closely related to the velocity and travel distance of the mechanical wave, but the relationship is the opposite of that of the light wave. The larger the mass density of the medium is, the faster the wave velocity of the mechanical wave propagated is and the longer the transmission distance is; conversely, the smaller the mass density of the medium is, the slower the wave velocity of the mechanical wave propagated is and the closer the transmission distance is. Mechanical waves of different wavelengths have different attenuation rates in the same medium.

Light travels at vastly different speeds in media with different mass densities. However, electromagnetic waves are thought to travel at the same speed whether they travel in a vacuum or in a conductor. Far infrared, for example, is a wave of light whose frequency is similar to that of high-frequency 
electromagnetic waves but whose physical properties are very different. In conductors, electromagnetic waves travel at the speed of light, and infrared rays travel very slowly.

\subsection{The experimental results of rotating the double-slit device in a specific direction show that the conclusion of "interference waves generate fringes" cannot be established}

As the double-slit device (Fig. 4(b)) rotates, as shown in Fig. 4(a), the arrangement direction of fringe La in the double-slit interference test results is always perpendicular to the midline $\mathrm{Lm}$ of the double slit. Obviously, water waves cannot show similar experimental results. There is no doubt that the direction of alignment (La) of water waves is always the horizon. This shows that the angle of the device rather than the light wave is the key factor determining the direction of the fringe arrangement. Only the light component sensitive to the $\mathrm{Lm}$ direction of the double slit or the slit angle through which the light passes is responsible for the striation. In any case, the conclusion that interfering waves generate fringes cannot be established.

\subsection{Interference conditions it is unacceptable to consider only frequency and phase and ignore the role of polarization direction}

As shown in Fig. 7, the vibration direction of S-wave is located on the normal plane $\mathrm{S}$ of its propagation direction. Water waves vibrate only vertically towards the center of the earth. The difference is that the direction of polarization of light is not just one but all over the plane $S$. The probability of the photon's polarization angle $a$ is evenly distributed in the range $0 \sim \pi$. Because it contains photons in all directions of polarization, a light beam like sunlight has no particular direction of polarization. Therefore, the conditions for the interference of two light waves include not only that the frequency of the two waves must be the same and the phase difference between them must be constant, but also that the polarization direction of the two waves must be the same. Because of polarization, light waves require far harsher interference conditions than water waves. For example, in order to watch a stereoscopic movie, two visual signals with horizontal and vertical polarization directions are projected on the screen at the same time, but the two signals do not interfere with each other, and the polarizing glasses can correctly separate the two clear light signals. Therefore, the stripes of the double-slit test are not caused by "interfering light waves".

\subsection{The phenomenon that reflected light does not interfere indicates that the conclusion of light wave interference is incorrect}

As shown in Fig. 8, reflected light waves can also cause interference. Objects can be seen because they reflect light. Screens are visible but not very reflective. However, the experimental results show that there is no interference effect of reflected light.

\subsection{Weak transmittance of double slit devices denies light waves}

Aiming or not, if light is a wave, it must pass through at least one slit. However, the experimental results show that the intensity of incident light is much greater than that of the stripe after transmission. At the 
same time, the light reflected by the double slit device is much more than the light transmitted. Only a small amount of light in the experiment managed to pass through the slit, which is just the light that travels through the slit. The vast majority of light that is not aimed at any slit cannot pass through the double slit device and is reflected or absorbed.

\subsection{The lithography machine will not work properly if the light waves passing through the slit interfere with each other.}

Both double-slit devices and masks have similar optical properties in their slits. According to the conclusion of the double-slit interference test, the light waves interfere with each other to produce fringes, then the mask will inevitably produce countless interference fringes due to the numerous slits, and it will be impossible for the lithography machine to reduce the patterns on the mask to the wafer clearly.

\subsection{Light waves cannot explain the distance travelled, although they can barely explain the different speeds of light}

As shown in Fig. 10, L1 and L3 have the same speed of light, and they have both speeds of light in air. The speed of light in L2 is the speed of light in glass, which is lower than the speed of light in air. This is only a far-fetched conclusion.

Another, more scientific interpretation: according to formula (1), the energy of a quantum is determined only by the frequency [1][2][3]. The energy of a photon is constant regardless of the medium it is in and is independent of its velocity. Thus, the density of the medium can slow down the light without partially depleting its energy. The density of glass slows down the speed of light but the energy of light passing through the glass remains the same.

Although the energy of a photon cannot be consumed in part, it can be destroyed as a whole. As the distance a photon travels through the medium increases, the photon is destroyed as a whole once the speed of light cannot be kept above the minimum threshold. If they travel a long enough distance, all the photons will be destroyed. So the denser the medium is, the slower the speed of light is and the shorter the distance it travels. The less dense the medium is, the faster the speed of light is and the longer the distance light travels. When light passes from a less dense medium to a more dense medium, the speed of light slows down. When light passes from a dense medium to a dense medium, the speed of light jumps.

\subsection{Different factors determine energy}

The factor determining the energy of electromagnetic waves is the amplitude, which has nothing to do with the frequency or wavelength, and because the amplitude is adjustable or limited, the energy of electromagnetic waves is adjustable. The amplitude of the electromagnetic wave can be partially cut off, which will generate an additional spectrum. Quantum theory[1][2][3] holds that the energy of quantum is determined by the frequency; it cannot be separated, and it can only be absorbed as a whole or remain 
unchanged. Therefore, its frequency cannot be changed during quantum existence; otherwise, it is another quantum.

\subsection{The absence of modulation effects by fiber optic communication technology indicates that light is not a wave}

In optical fiber communications[15][16][17], the transmitted digital signal has a spectrum base band whose bandwidth is proportional to the communication rate. Theoretically, the spectrum bandwidth of the digital signal is infinite. If light is a wave, it is a carrier. Without loss of generality, let the carrier be $\sin \left(\omega_{c} t\right)$ and the digital signal be $\cos \left(\omega_{s} t\right)$. Then, $\omega_{c}$ is a single-frequency constant, and $\omega_{s}$ is not a singlefrequency constant but a range. The modulation results:

$s(t)=\sin \left(\omega_{c} t\right) \cos \left(\omega_{s} t\right)=\left[\sin \left(\omega_{c}+\omega_{s}\right) t+\sin \left(\omega_{c}-\omega_{s}\right) t\right] / 2$

The resulting spectrum is a conjugate spectrum of $\omega_{c}+\omega_{s}$ and $\omega_{c}-\omega_{s}$ centered on the carrier frequency $\omega_{c}$ This means that the frequency of the photon is changed from one to two conjugated spectra of the same width as the base band of the signal. Since photons of different frequencies are different photons, modulation changes photons of one frequency into a series of photons of different frequencies. This obviously violates the fundamental principles of quantum mechanics, and in fact, it does not happen in fiber-optic communications. Moreover, the spectrum in the baseband is continuous, but not every frequency spectrum exists.

\subsection{The light wave model is inconsistent with the widely used Fourier transform principle}

Quantum mechanics states that any quantum has only one frequency and that different frequencies point to different quanta. In real life, lasers have only one frequency. That is, the spectrum of the quantum and laser has only one line and no bandwidth. However, although it is a basic physical parameter of wave frequency, according to the Jean Baptiste Joseph Fourier transform principle[12][13][14], because of the limited time and space of waves at both the starting point and end point, start or finish must undergo a transition process to reach steady state, so no waves exist only in the limited time, and space waves may be only one frequency and must be a band. This conclusion has been confirmed by wireless communications technology[10][11]. Otherwise, if there were zero bandwidth electromagnetic waves, the glorious achievements of radio communication technology would not be worth mentioning.

\subsection{The form of magnetic induction line is only applicable to electromagnetic waves}

Magnetic inductance lines are closed loops, and no corresponding physical form can be found in the light.

\subsection{Light waves cannot be focused}

Waves propagate infinitely in all directions. If light is a wave, it does not explain the focusing principle. 


\subsection{A photon is equivalent to a sample of a light wave, the base of which collapses}

If light is a wave, the diameter of the photon is much smaller than its wavelength. Because photons occupy space indiscriminately, the number of photons that can be arranged within a single wavelength is huge. In the diagram below, shown in Fig. 11, a single photon occupies only a negligible fraction of the total period. Therefore, a photon is equivalent to only one sample of the light wave, and the sampling frequency $f_{s}$ is the product of the basic frequency $f$ of the photon and the sampling number, which is the ratio of wavelength to photon size. The spectrum of photon is the sampling spectrum, which contains abundant frequencies. Only smooth waveforms have the fewest harmonics, so the photons that make up a cycle must be a set whose members have the same fundamental frequency. If the phase difference between the photons is constant $\delta$, then the phases of the photons are $k \delta$, and $k$ is an integer. The order of photons in the light wave is $k>0$, and $k$ increases from small to large. The phase difference between adjacent photons is $\delta$. The photons are arranged in phase order. When a photon moves, its phase does not change with time.

\subsection{Neither of the known models is justify}

Newton's particle model was rejected because the double-slit test showed alternating streaks of light and dark. However, wave-particle duality [4][5] is also unscientific. In any case, the wave model is a misconception. As shown in Fig. 9, medium $A$ is above the interface, the speed of light is $V_{A}$ and the wavelength is $\lambda_{A}$. Below the interface is medium $B$, the speed of light is $V_{B}$, and the wavelength is $\lambda_{B}$. According to Huygens' principle, what causes refraction when light passes through the interfaces of different media is that light's refractive index is the ratio of wavelengths in different media. Light waves have different wavelengths because of the speed of light in different media. Notice that the speed of light is the fastest and the wavelength is the longest in vacuum. However, this principle is open to question. Light is not a wave, and the key factor that causes refraction is the difference in the speed of light in different media.

As shown in Fig. 5, according to Thomas Young's wave interference principle, only the waves generated from the wavelet source generated by the same wave source can meet the conditions of interference. After passing through gap $a$ of single-slit device $\mathrm{S1}$, a light wave reaches gap $b$ and $c$ of double-slit device $\mathrm{S} 2$ at the same time and becomes two coherent light waves. After they interfere with each other, the stripes in the double-slit test result are generated. The principle holds that light is always a wave. However, the medium through which water waves are transmitted is fluid, and if there are no obstacles the waves will travel smoothly through the medium. Otherwise, when water waves encounter a hard enough barrier, their normal propagation path is blocked and new wavelet sources are created at the barrier. Because the energy of a water wave is evenly distributed on the wave front, a water wave encountering an obstacle will reflect a wavelet with the same frequency, wavelength, and velocity as the source, but with a phase difference of $\pi$. A wavelet source is generated in the stripes and water waves from different wavelet sources can interfere at the intersection point. Note that the energy of water wave is transmitted by means of medium water. When the normal propagation path is blocked, water wave is 
converted into wavelet source to meet the law of energy conservation. That is, the double slit interference of water wave completely obeys the relevant physical laws.

The propagation of light requires no medium. The light source is the radiation phenomenon of electron energy level transition. In practice, there is no experimental support for the formation of a new sub-light source in the slit. This is because the double-slit experiment can be done in a vacuum without any material, where there is no material required to produce radiation in the slit, and the sub-light source is generated from nothing. And because different substances radiate light of different physical properties, it is impossible for the sub-source in the slit to radiate the same wave of light as the source. In addition, since all light from a light source spreads from the source, if a new light wave is created at the slit, it cannot travel in the same direction as the light source and nothing else.

\subsection{Two startling conclusions are inescapable}

According to Doppler's principle, the movement of a wave source towards the observer is said to proceed and the wavelength is compressed. Instead, the wave source moving away from the observer is called retrogression, and the wavelength is stretched. According to this principle, when light is a wave, the spectrum of the star will be blue shifted and when it is retrograde, the spectrum of the star will be red shifted. Thus, in a binary star system orbiting each other, if the stars are exactly at opposite ends of their orbits relative to the Earth, one of them will move toward the Earth and the other away from it. Stars that move toward Earth are called progress. stars that move away from Earth are called regressions. If light is a wave, according to Doppler's principle, the fading star must undergo a redshift and the advancing star must undergo a blueshift. However, observations showed that the stars in the binary system did not change color. One of the most plausible explanations is that because light is not a wave, it does not have the conditions necessary to produce the Doppler effect, so the colors of the stars in a binary star system do not change. But the light in this phenomenon is still forced to be interpreted as a wave, which leads to two startling conclusions:

Problem 1: There is no redshift or blueshift because the speed of light is constant and independent of the frame of reference. This conclusion is the cornerstone of relativity. Obviously there is no theoretical basis for this hypothesis.

Problem 2: Completely ignoring the objective fact that the color of stars in a binary star system remains unchanged, Hubble's law that the farther away from the Earth the more serious the redshift of celestial bodies is is forcefully derived [6][7]. The constant color of stars in both retrogression and dual star system means that the phenomena of redshift and blueshift are impossible. Then the redshift required by Hubble's law would not have occurred. In addition, the derivative conclusions of Hubble's law include: the expansion of the universe, the Big Bang, dark matter and dark energy. However, concepts such as dark matter conflict with Kepler's planetary laws [8][9]. In fact, Kepler's three laws of planets stand up to all the celestial bodies in the solar system. Uranus and Neptune were found using these three laws in the absence of dark matter and dark energy. If we add dark matter and dark energy, our solar system won't exist. 


\subsection{The phenomenon of "observation determines outcome" has also been observed}

In the causality test of double slit interference. Test 1: Before the introduction of observation, the test result was multiple stripes alternating between light and dark, as shown in Fig. 12(a). In this case the conclusion is that light is waves, interfering waves that produce interference fringes. Test 2: If observation is introduced, there are only two bright stripes with a dark stripe in the middle, as shown in Fig. 12(b). In this case the conclusion is that light is a particle and no interference of light waves has occurred. The cause of the two different test results was identified as whether or not they were observed. If so, it leads to the following confusing logic: It is also observation that knows the phenomenon that observation changes the result of an experiment. Both interference phenomena without introduction of observation and particle phenomena with introduction of observation are observed. Whether the observer participates in the experiment or not, the results of the experiment will be observed eventually. If being observed can affect the outcome of the experiment, can the final observation also affect the outcome of the observed influence experiment? If instrumental observation causes the wave-particle duality to collapse into particles, and human observation does not cause the wave-particle duality to collapse, how can photons distinguish observers, can the instrument not be observed? If being observed makes a difference, then why must the collapse rules be followed: when not observed, they collapse only as waves and when observed, they collapse only as particles and not the other way around? If the light which has collapsed into particles is projected onto another double-slit device as a light source, can "interference" fringes be generated again?

\section{Conclusion}

In conclusion, light waves, the wave-particle duality of light, and Newton's particle model of light are all wrong. However, the new quantum model must agree with the results of the double slit test. So Thomas Young's double slit experiment was still a great physical experiment.

\section{Instructions}

\subsection{The physical significance of quantum parameters such as frequency, wavelength and period}

Although not a wave, a quantum still has physical parameters such as wavelength, frequency and period. The quantum objects described by these parameters include: transition period, size fluctuation and so on. The size of quanta may fluctuate periodically. Although the energy of a quantum is fixed, the spatial distribution of its energy density may fluctuate periodically.

\subsection{The light}

Just as current is a directional flow of charge, light is a flow of photons. In any case, light never expresses the properties of waves. All the conclusions about light waves are a distortion of the relevant experimental results. 


\subsection{There is no collision or energy exchange between intersecting rays}

Since photons have a rest mass of zero, they are transparent to each other, and any photon does not prevent other photons from using the space they already occupy. There is no limit to the degree of overlap between the spaces occupied by different quanta, and quantum theory also holds that multiple photons can occupy the same space at the same time. As a result, nothing happens to the intersecting photons when they collide, neither exchanging energy nor changing the direction of any of the photons. This rule only applies to photons with zero rest mass on both sides, otherwise, when contact occurs, the object can change the direction of the photon's motion or make it disappear.

\section{References}

1. Quantum Mechanics, Yin Hongjun, University of Science and Technology of China Press, October, 1999.

2. Quantum Physics, Zhao Kaihua, Luo Weiyin, Higher Education Press

3. Feynman, Richard. Leighton, Rob; Sandoz, Matthew Feynman's lecture on physics III (1) quantum behavior. Taiwan: the world culture book. 2006.

4. P. Mittelstaedt, A. Prieur and R. Schieder, Unsharp particle-wave duality in a photon split-beam experiment, Foundations of Physics, 17, 891-903 (1987) .

5. Greene, Brian. The Elegant Universe: Superstrings, Hidden Dimensions, and the Quest for the Ultimate Theory. New York: W.W.Norton. 1999: pp.97-109.

6. Hubble, Edwin, "A Relation between Distance and Radial Velocity among Extra-Galactic Nebulae" (1929) Proceedings of the National Academy of Sciences of the United States of America, Volume 15, Issue 3, pp. 168-173

7. Move over, Hubble: Discovery of expanding cosmos assigned to little-known Belgian astronomerpriest | Science | AAAS

8. Johannes Kepler, Harmony in the World, Peking University Press, 2011

9. David Yang, Lectures on General Astronomy, August 1999

10. Fan Changxin, Cao Lina, Principle of Communication (7th Edition), National Defense Industry Press, September 2013

11. Jiongban-Zhou, Communication Principles (3rd Edition), Beijing University of Posts and Telecommunications Press, August 1, 2008

12. C. C. Lin \& L. A. Segel, Mathematics Applied to Deterministic Problems in the Natural Sciences, Macmillan Inc., New York. Beijing, Science Press, 1974

13. The Scientist and Engineer's Guide to Digital Signal Processing $₫ 1997-09-09$

14. Li Wenke, The Magic Matrix Season 2, Harbin Engineering University, 2014

15. Zhang Baofu. Optical Fiber Communication: Xidian University Press, 2009-09 
16. Zhang Hongbin, Qiu Kun, Zhou Dong. Wavelength Division Multiplexing Optical Fiber Communication Technology: 2000-04, Journal of University of Electronic Science and Technology of China

17. Liu Xilian, Peng Tianxiang. Optics and Soliton Communication: Physics and Engineering, 2002-05

Figures

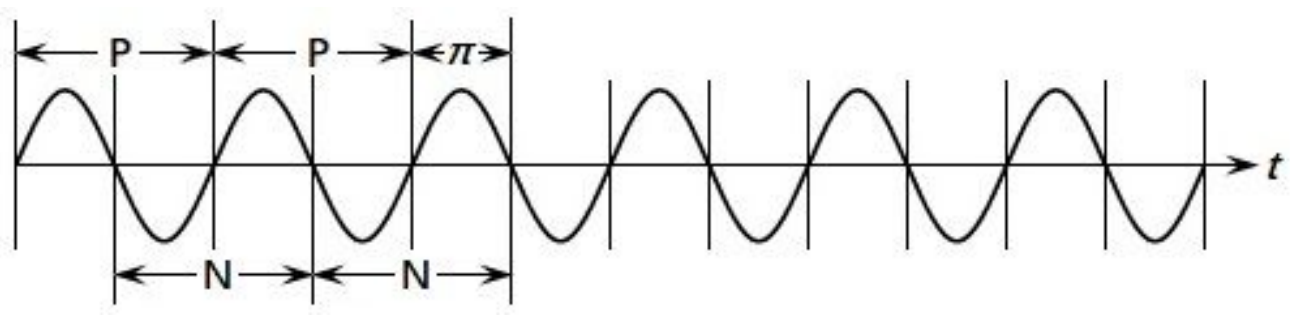

\section{Figure 1}

Matter and antimatter waves

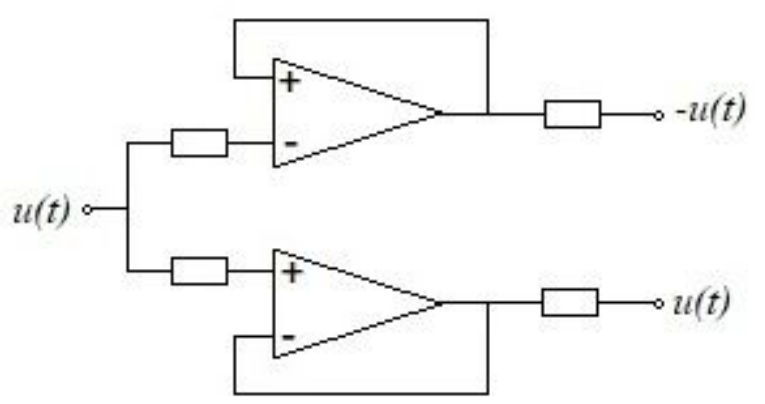

\section{Figure 2}

Circuit for generating mutually inverting signals 


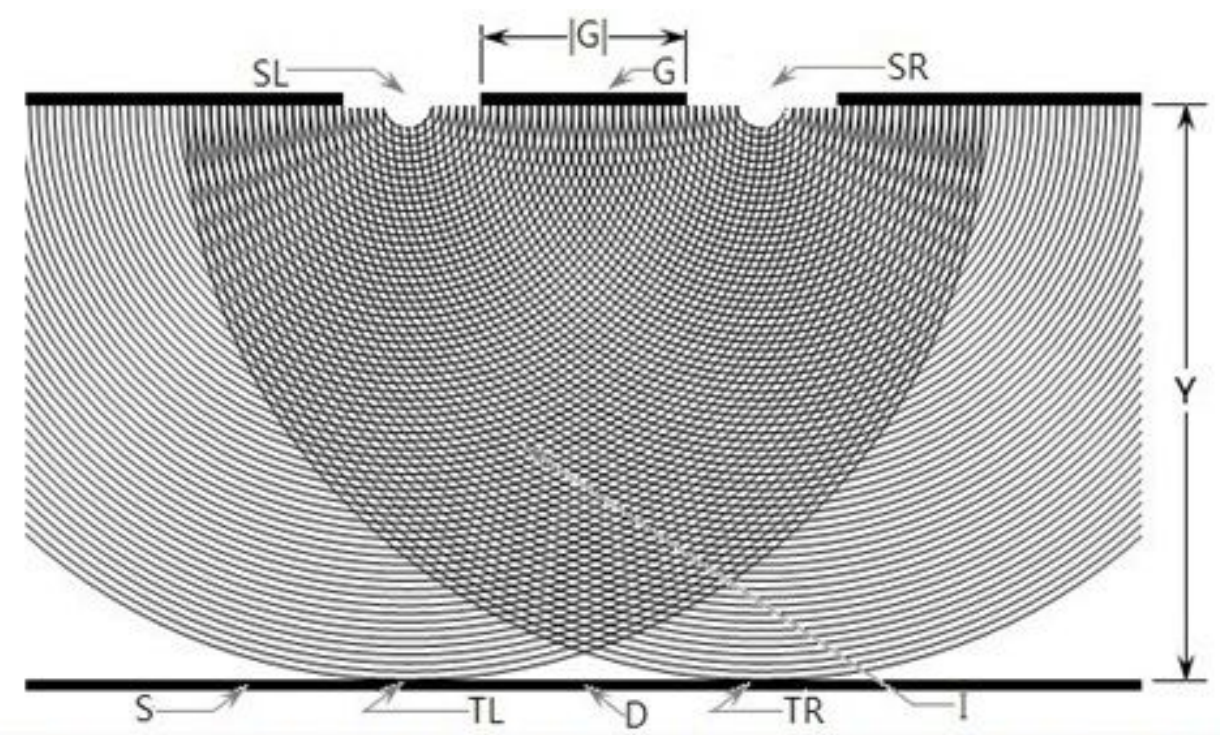

G: The space between two slits. It is located between two slits on the double-slit device. The width is recorded as $|\mathrm{G}|$. S: Screen. Where the light is imaged. I: Interference area. Y: The distance between the double-slit device and the screen.SL: left slit on double-slit device.SR: right slit on double-slit device. TL: Left target in double-slit test, the contact point of left wave surface on the screen.TR: Right target in a double-slit test, the contact point of the right wave surface on the screen. D: The midpoint between the left and right targets.

\section{Figure 3}

Schematic diagram of the double-slit interference test principle
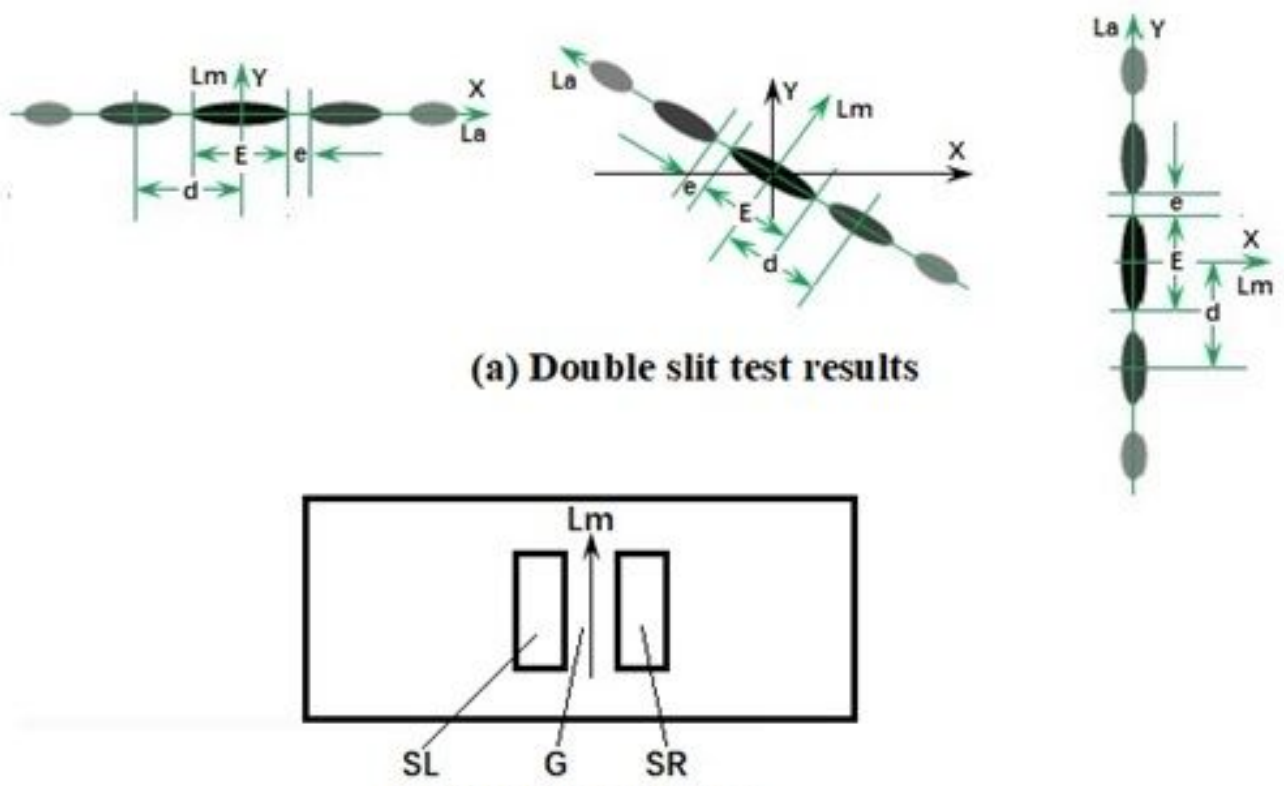

(b) Double-slit device

X: Horizontal direction. Y: Vertical direction. Lm: double-seam center line. La: the arrangement direction of stripes, $\mathrm{La}$ is perpendicular to $\mathrm{Lm}$. d: Fringe spacing. $\mathrm{E}$ : The width of the bright stripe. e: The width of the dark stripe. SL: Left slit. SR: Right slit. G: the gap between the two slits, width for $|\mathrm{g}|$. Width of slit is $|\mathrm{g}|$, Height of slit is $|\mathrm{h}|$. 
Double slit test results and device

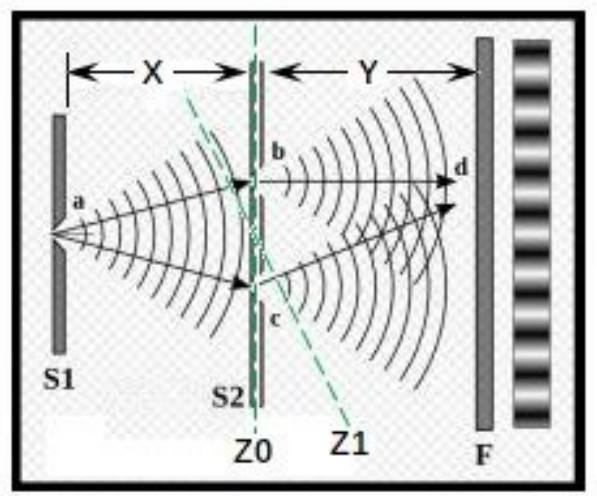

Figure 5

Principle of double-slit interference

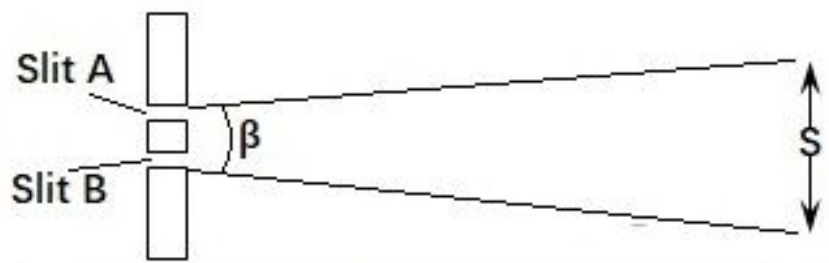

$\beta$ : The divergence angle of double-slit test results.

$\mathrm{S}$ : The range of fringes in double-slit test results.

\section{Figure 6}

divergence angle of double-slit test results

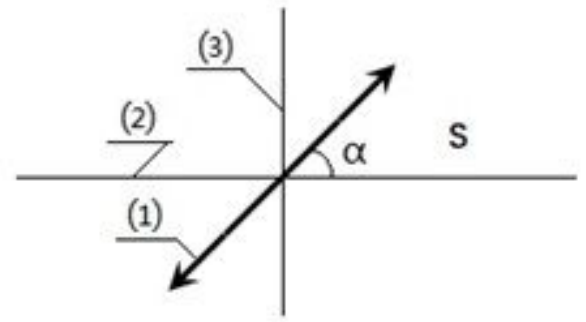

$\mathrm{S}$ : The normal plane of the direction of light propagation

(1)The polarization direction of photon.

(2) Horizontal direction, which is perpendicular to the propagation direction of light.

(3) Vertical direction, which points to the center of the earth.

$\alpha$ : The polarization direction of the photon, which is the angle between the vibration direction of the photon and the horizontal direction (2).

\section{Figure 7}

A cross section diagram of the horizontal path of a photon 


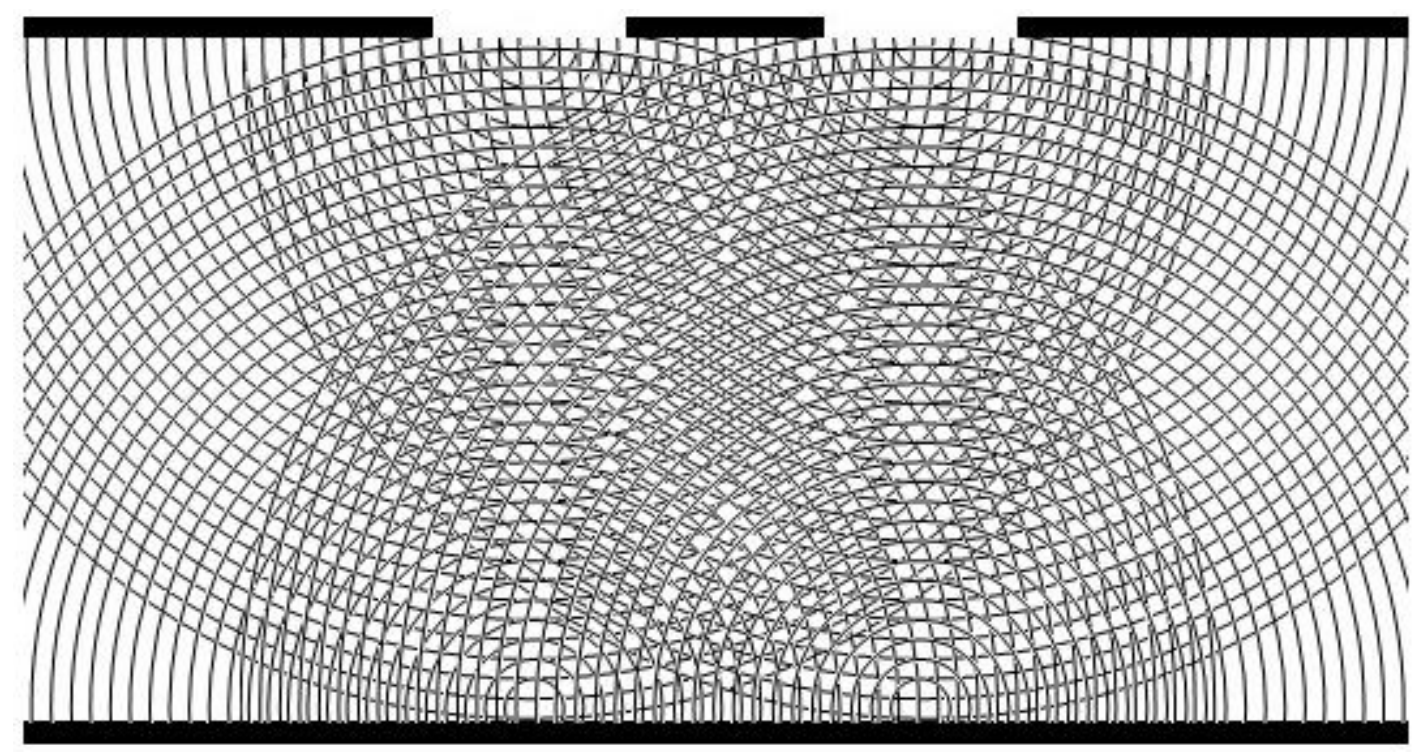

Figure 8

Interference from the light reflected by the screen

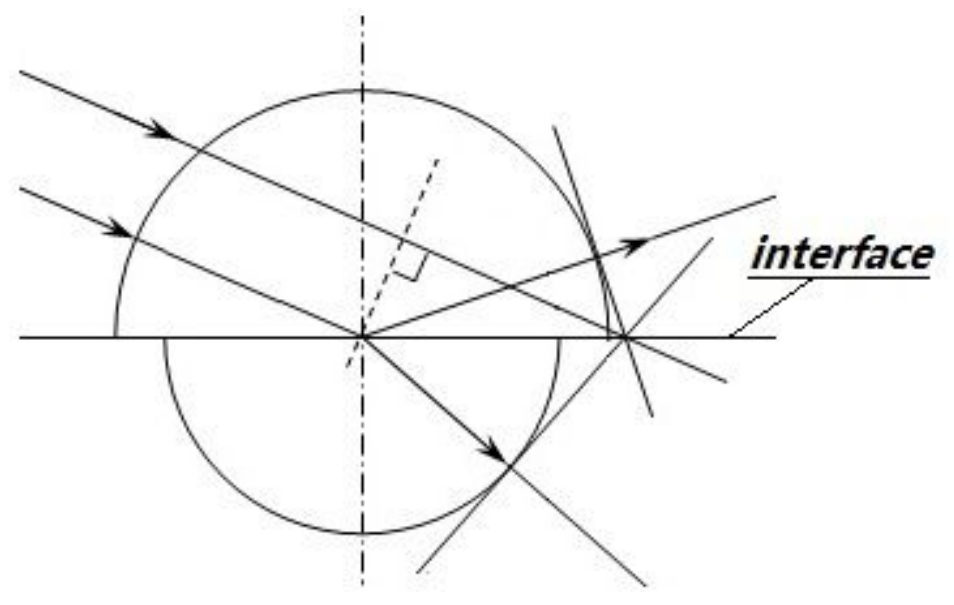

Figure 9

Refraction of light, Huygens' principle

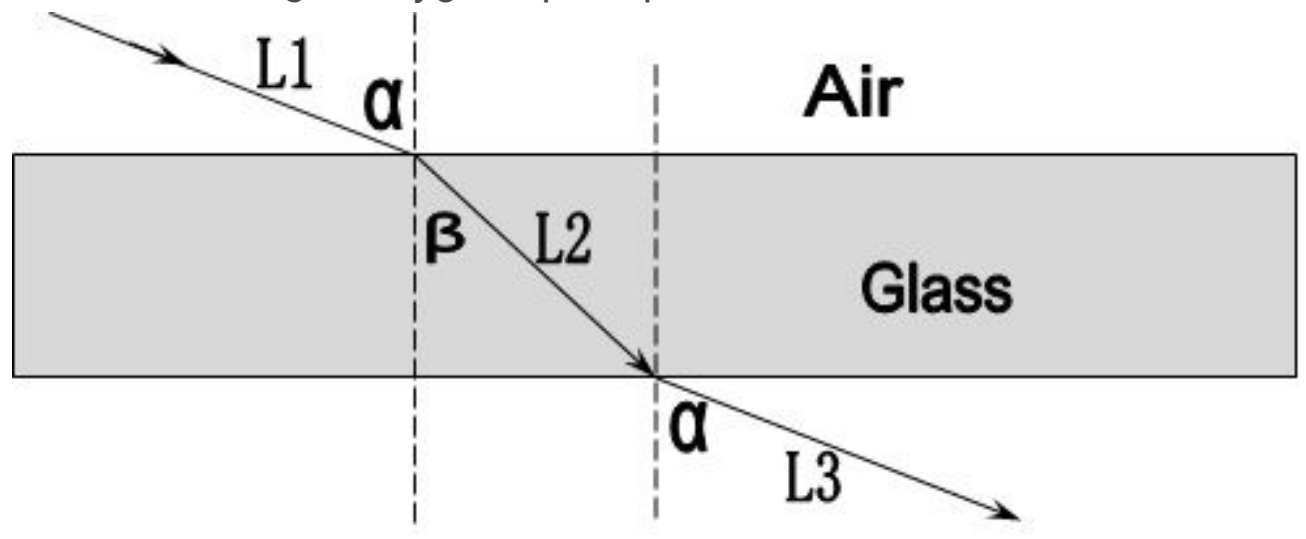

Figure 10 
Light passing through glass

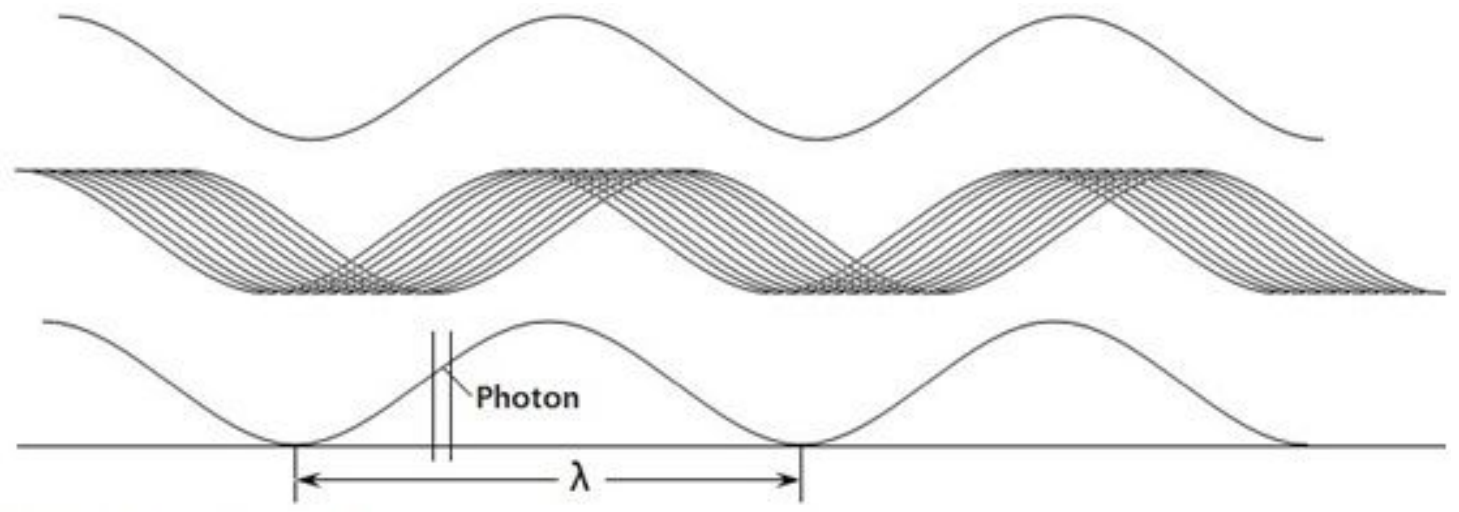

Above image: Single light wave

Middle image: Multiple light waves in different phases

Below image: Size relationship between photons and light wave period

\section{Figure 11}

Light waves

(a)

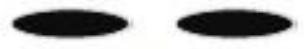

(b)

(a) Test results that do not introduce observations

(b) The experimental results after the introduction of observations

\section{Figure 12}

Double slit test results with or without observation 